\title{
Redundancy Reduction for Compressed Sensing based Random Equivalent Sampling Signal Reconstruction
}

\author{
${ }^{*}$ Jianguo Huang, Li Wang and Yijiu Zhao \\ School of Automation Engineering, University of Electronic Science and \\ Technology of China, Chengdu, 611731, China \\ E-mail:jghuang@hotmail.com
}

\begin{abstract}
Random equivalent sampling (RES) can composite a waveform with high equivalent sampling rate from multiple low speed sampling sequences. In practical application, the performance of RES signal reconstruction would be degraded by the non-uniform distribution of sampling time. Compressed sensing (CS) theory is adopted to reconstruct RES samples, which could mitigate the inherent coherence of sampling time. However, the CS reconstruction algorithm is sensitive to the signal sparsity level that is unknown in the reconstruction stage. In this paper, we propose a redundancy reduction algorithm for CS base RES signal reconstruction that can ensure reconstruction accuracy while reducing the number of random samples. The experimental results are reported to evaluate the performance of the proposed algorithm.
\end{abstract}

Keywords: random equivalent sampling, compressed sensing, redundancy reduction, signal reconstruction

\section{Introduction}

Signal sampling, as a bridge from analog domain to digital domain, is the basis for the digital signal processing theory. Analog to digital converter (ADC) is the fundamental way to capture the analog signal. With the development of information technology, signal with very high frequency is fed into digital system, Such as communication systems or radar systems, the signal frequency exceeds $1 \mathrm{GHz}$. Governed by the famous Shannon sampling theory, the sampling rate of ADC should be two times of signal frequency. ADC technology cannot satisfy the requirements of this felids. In order to address this challenge, many alternative sampling techniques are proposed.

In the instrument applications, the time interleaved sampling [1-2] and random equivalent sampling (RES) [3-4] are wildly used. In time interleaved sampling, multiple parallel ADCs are used to capture a signal. One can realize the time alternate sampling for the signal by controlling the sampling clock phase. Time interleaved sampling could be cost effective or even only possible solution in sampling non-repetitive signal due to the high cost or unavailable of a single ADC solution. The circuit realization of time interleaved sampling technique is complex, Since ADCs are asynchronously clocked [5], the non-uniform error would be introduced. In many practical applications, signal may be repetitive. RES would be an attractive sampling technique. RES samples a high frequency periodic analog waveform at a sub-Nyquist rate using a single analog-to-digital converter (ADC) clocked at a lower frequency. It assumes the analog waveform may be triggered no more than once per period at exactly the same relative position. Leveraging the insynchrony between the sampling clock and the triggering signal, each time a sample is taken at a different position of a cycle of the analog waveform. A time-to-digital converter (TDC) [6] measures the time offset between the triggering point and the sampling clock

${ }^{*}$ Correponding Author 
edge and aligns the time samples at approximately correct positions within a period of the periodic analog waveform. The analogy waveform then may be reconstructed using these interleaved time samples at higher resolution.

Very often, RES takes considerable longer than competing time interleaved sampling methods which use multiple ADCs in parallel. This is because after each sample is taken, the time offset will have to be measured by TDC to time-align the time sample within a period of the analog waveform. Moreover, the relative sampling positions within a period may not be evenly distributed. To achieve desired accuracy, it may take significantly longer time to perform RES.

Compressed sensing (CS) [7-9] is a new signal processing theory, which can reconstruct signal from a small number of low speed samples. CS algorithm has been introduced to RES signal reconstruction (called CS-RES) [10]. Compared to the traditional time-aligned RES signal reconstruction algorithm, the CS-based reconstruction algorithm can significantly improve the efficiency of RES. Generally, the reconstruction precision of CS algorithm is sensitive to the underlying signal sparsity level. In the signal reconstruction stage, the prior knowledge of signal sparsity level is unavailable. In order to achieve desirable reconstruction accuracy, one has to capture enough RES acquisitions.

In this paper, we present an algorithm as the stopping criteria for CS based RES signal reconstruction. Different from the traditional sequential compressive sampling judgment criteria (SCSJC) [11], which is based on comparison between the current reconstruction and the previous reconstruction, the proposed algorithm is based on the coherence test of samples. With the help of proposed algorithm, the redundancy of samples could be reduced, and a reasonable acquisition number could be obtained.

In the remaining of this paper, the RES sampling method is reviewed, and the CS based RES signal reconstruction algorithm is briefly reviewed in section 2 . The redundancy reduction algorithm is proposed in section 3. The orthogonal matching pursuit algorithm is introduced in section 4 . The experimental results are reported in section 5. The paper is concluded in section 6 .

\section{Random Equivalent Sampling}

\subsection{Fundament of Random Equivalent Sampling}

The basic principle of RES sampling is illustrated in Figure 1. Given a repetitive signal as shown in the solid line on the top row, a level-trigger circuitry compares this analog waveform against a reference voltage shown as the horizontal dashed line. A trigger pulse will be generated whenever the voltage of the analog signal rises crossing the reference voltage. A sample is taken by the immediately sampling pulse after the trigger pulse. It is assumed that exactly one trigger pulse will be generated in each acquisition. The trigger pulse also provides a fixed reference point to align samples. $\Delta t_{m}\left(0 \leq \Delta t_{m} \leq T_{s}\right.$, and $T_{s}$ is the sampling interval $)$ is the relative sampled position of the $m$ 'th sampled signal within a period of the sampling clock. A waveform with high equivalent sampling frequency can be aligned from lowspeed samples.

In the reconstruction stage of RES, the sampling interval $T_{s}$ is partitioned into equally spaced bins of duration $T_{e}$. The value $f_{e}=1 / T_{e}$ then becomes the equivalent sampling frequency of RES. The value of $T_{e}$ should be chosen to be $<T_{0} / 2$ so that the equivalent sampling rate meets the requirement of Shannon sampling theorem ( $T_{0}$ is the period of underlying signal). 


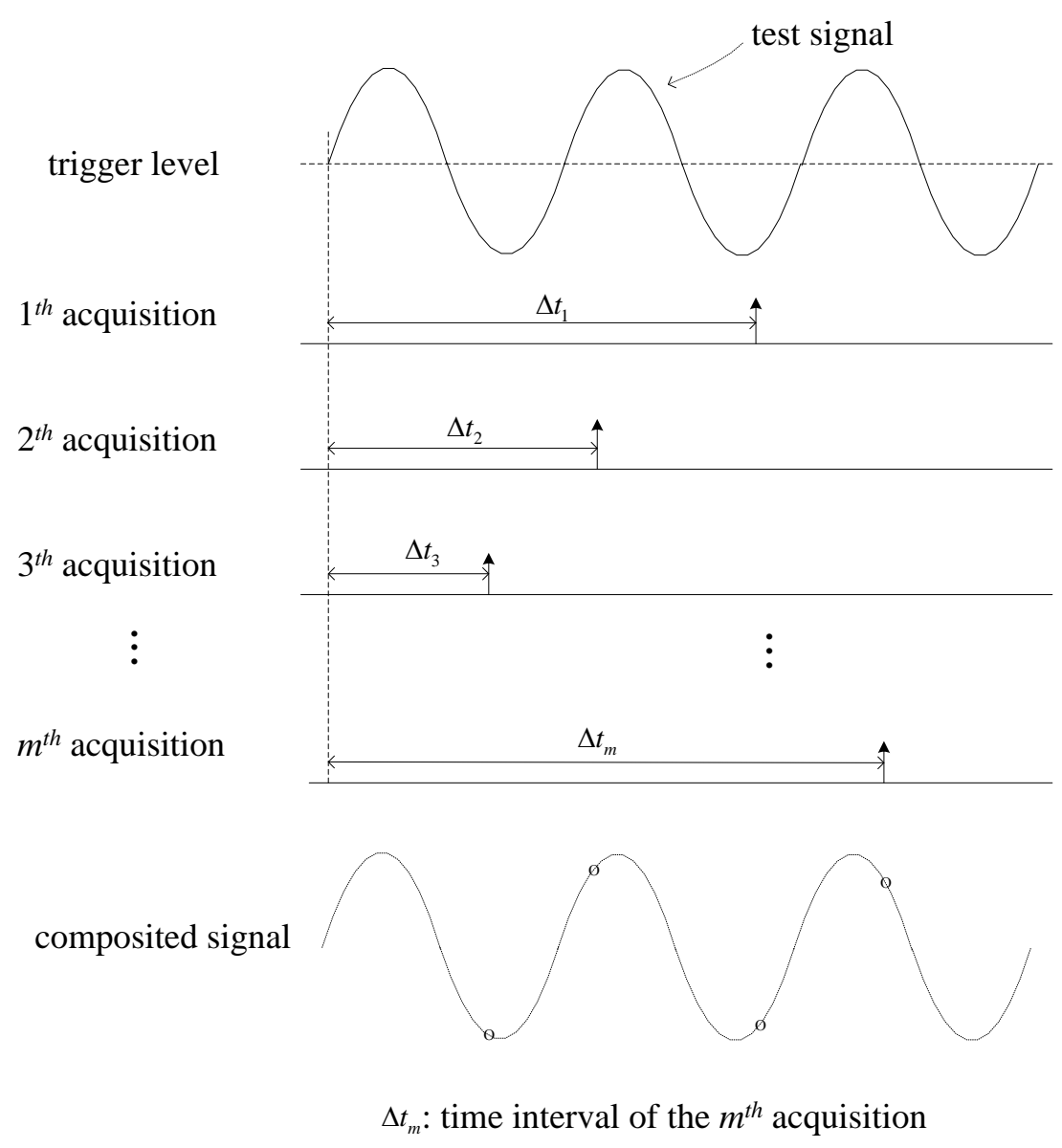

Figure 1. The Scheme of Random Equivalent Sampling

According to RES, the time interval $\Delta t_{m}$ is the duration between the trigger signal and the rising edge of the immediately following sampling clock, and $\Delta t_{m}$ distributes in the interval $\left[0, T_{s}\right]$. For an $N$ dimensional reconstructed signal, the farthest distance between the equivalent sample and the trigger signal is $N \cdot T_{e}$. So reconstructed signal length $N$, sampling clock period $T_{s}$, and equivalent sampling period $T_{e}$ need to satisfy relation: $N=T_{s} / T_{e}$.

Due to the uneven sampled position, some bins may have more than one sample falling into them while some bins may be left unfilled for a long duration, and RES would require a rather large number of samples to fill the empty bins and thereby achieve the desired accuracy. Clearly, the inefficiency of the RES sampling method is demonstrated.

In order to measure time interval, time interval needs to be expanded. Time intervals are expanded through the fast charging circuit and slow discharging circuit, which is called TDC. As described in Figure 2, the trigger pulse starts the charging circuit, and then the capacitor is charged with the large constant current, the first sampling clock after the trigger pulse stops the charging process and at the same time the capacitor is discharged with a small constant current. The charging time $t_{c}$ is very short, and on the contrary, the discharging time $t_{d}$ is long. The counter circuit counts $t_{c}$ and $t_{d}$, which are the expanded time of the time interval between the trigger point and the first sample clock edge after trigger. 


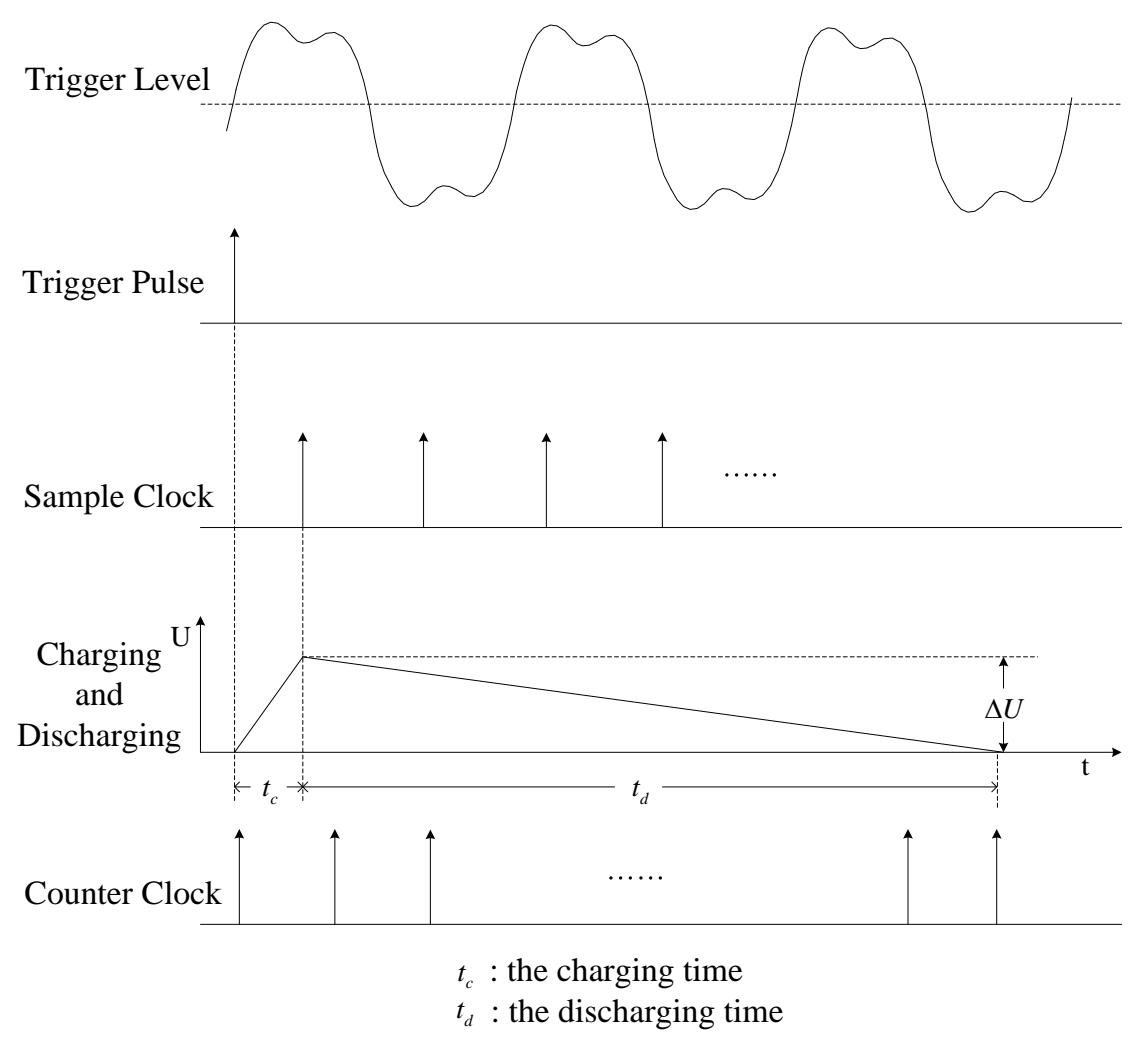

Figure 2. The Principle of TDC. Time Intervals are Measured Indirectly after Charge-Discharge Process

After the charge-discharge process, the voltage variation on the capacitor is zero. Therefore, we can have the equation as follow:

$\Delta U=\frac{I_{c} \cdot t_{c}}{c}=\frac{I_{d} \cdot t_{d}}{c}$.

where $c$ is the capacity of the capacitor, $I_{c}$ and $I_{d}$ are the charging current and the discharging current respectively $\left(I_{c}>>I_{d}\right)$. When $I_{c}$ and $I_{d}$ are constants, $t_{d}$ is proportional to $t_{c}$. According to (1), we can have $R=t_{d} / t_{c}=I_{c} / I_{d}$, we can control $R$ through changing $I_{c}$ and $I_{d}$.

Time interval $\Delta t_{m}$ (or $t_{c}$ in Figure 2) is uniformly distributed in the interval $\left[0, T_{s}\right]$, which may be very small. However, if $\Delta t_{m}$ is very close to " 0 ", the TDC will not work properly. In practice, time interval $\Delta t_{m}$ is measured after superimposed a time interval $T_{c}$ $/ 2\left(T_{c}\right.$ is the period of the counter clock, and let $T_{c}$ equal to $\left.T_{s}\right)$, and then $t_{c}$ is uniformly distributed in the interval $\left[T_{c} / 2,3 T_{c} / 2\right]$.

The measurement precision of time interval affects the accuracy of signal reconstruction directly. However, the TDC circuit always subjects to the temperature, and it is difficult to measure $t_{d}$ accurately. In order to eliminate the effect of $t_{d}$ on the measurement precision, the measurement process should be carried out carefully. In practical application, the time interval is measured with three steps. Let $t_{1}=T_{c} / 2, t_{2}=$ $3 T_{c} / 2$. First, measure $t_{1}$ and $t_{2}$, and then measure $\Delta t_{m}+T_{d} / 2$, the measuring steps are described as follows:

(a) when $t_{c}=t_{1}$, after charge-discharge process, $K_{1} T_{c}=t_{c}+t_{d}=(R+1) t_{1}$ and count value $K_{1}$ can be rewritten as:

$K_{1}=(R+1) / 2$. 
(b) when $t_{c}=t_{2}$, after charge-discharge process, $K_{2} T_{c}=t_{c}+t_{d}=(R+1) t_{2}$ and count value $K_{2}$ can be rewritten as:

$K_{2}=3(R+1) / 2$.

(c) when $t_{c}=\Delta t_{m}+T_{c} / 2$, after charging and discharging, $K_{m} T_{c}=t_{c}+t_{d}=(R+1)\left(\Delta t_{m}\right.$ $+T_{c} / 2$ ), and the count value $K_{m}$ can be rewritten as:

$K_{m}=(R+1)\left(\Delta t_{m}+T_{c} / 2\right) / T_{c}$.

From (2)-(4), time interval $\Delta t_{m}$ can be rewritten as:

$\Delta t_{m}=\frac{K_{m}-K_{1}}{K_{2}-K_{1}} T_{c}$.

Therefore, $\Delta t_{m}$ has nothing to do with $R$. In other words, through calibration, we can avoid the impact of temperature on the TDC.

\subsection{CS based Signal Reconstruction}

Compressive sampling (CS) signal reconstruction algorithm can successfully reconstruct the original signal from a small number of low-speed random sampling values. In RES, signal to be captured is required to be repetitive that is sparse in its Fourier transform domain. It is consistent with the requirement of CS. Naturally, CS is used to improve the RES signal reconstruction [10].

In CS, the signal to be reconstructed is denoted by an $N$ dimensional vector $\mathbf{x}$. In the current application, $\mathbf{x}$ would be the unknown analog signal sampled at Nyquist rate over the time duration $T_{e}$. Using RES or other methods, a set of measurements of the elements of $\mathbf{x}$ is obtained and denoted by a vector $\mathbf{y}$. In RES, each element of $\mathbf{y}$ is the output of a low-rate ADC. It can be represented as a weighted linear combination of elements in $\mathbf{x}$ over the sampling window $T_{e}$. Let these weights be arranged in a measurement matrix $\boldsymbol{\Phi}$, one may express the relation between $\mathbf{x}$ and $\mathbf{y}$ as follows:

$\mathbf{y}=\Phi \mathbf{x}$

Since the analog signal is periodic, it admits a Fourier series expansion representation:

$$
\begin{aligned}
x(t) & =\sum_{n=-\infty}^{\infty} \alpha_{n} \exp \left(\frac{j 2 \pi n t}{T_{0}}\right) \\
\alpha_{n} & =\frac{1}{T_{0}} \int_{t=0}^{T_{0}} x(t) \exp \left(-\frac{j 2 \pi n t}{T_{0}}\right) d t
\end{aligned}
$$

where $\left\{\alpha_{n}\right\}$ are Fourier coefficients. A signal is spectrally sparse if only very few Fourier coefficients have significant magnitudes while other Fourier coefficients are nearly zero. In other words, the energy of the signal is concentrated on few spectral coefficients. For the signal $\mathbf{x}$ with $K$ significant spectral coefficients, we call it $K$-sparse signal, and $K$ is sparsity level that is usually unknown in the signal reconstruction stage. Since $x(t)$ is band-limited in practice, the signal $\mathbf{x}$ may be represented as:

$\mathbf{x}=\Psi \alpha$

where $\alpha$ is a sparse vector consisting of few non-zero Fourier coefficients and $\Psi$ is the Fourier basis, which is approximated by the Discrete Fourier Transform (DFT) basis, and $\psi_{i, k}=\exp (-j 2 \pi i k / N) / \operatorname{sqrt}(N), \quad 1 \leq i, k \leq N$ [12]. Combining (6) and (8), one has

$\mathbf{y}=\Phi \mathbf{x}=\Phi \cdot \Psi \alpha$. 
Considering the practical situation of RES sampling, the uneven distribution of sampling time intervals makes RES technique inefficient. With the help of CS reconstruction algorithm, one only needs a small number of RES samples. In CS signal reconstruction stage, we need to construct the mathematical model of RES. For CS-RES signal reconstruction, measurement matrix based on Shannon interpolation formula is constructed.

$$
\begin{array}{r}
{\left[\begin{array}{c}
y\left(\Delta t_{1}\right) \\
y\left(\Delta t_{2}\right) \\
\vdots \\
y\left(\Delta t_{M}\right)
\end{array}\right]=\left[\begin{array}{cccc}
\phi_{1,1} & \phi_{1,2} & \cdots & \phi_{1, N} \\
\phi_{2,1} & \phi_{2,2} & \cdots & \phi_{2, N} \\
\vdots & \vdots & \ddots & \vdots \\
\phi_{M, 1} & \phi_{M, 2} & \cdots & \phi_{M, N}
\end{array}\right]\left[\begin{array}{c}
x\left(T_{e}\right) \\
x\left(2 T_{e}\right) \\
\vdots \\
x\left(N \cdot T_{e}\right)
\end{array}\right]} \\
\mathbf{y}=\mathbf{\Phi \mathbf { x }}
\end{array}
$$

where

$\phi_{m, n}=\operatorname{sinc}\left(\frac{\Delta t_{m}}{T_{e}}-n\right)$

where $\mathbf{y}$ is the RES sampling sequence, $\mathbf{x}$ is the Nyquist sampling sequence that is to be reconstructed.

From (10), we can compute the measurement matrix $\Phi$ according to the sampling time interval $\Delta t_{m}$. Given the measurements $\mathbf{y}$, the measurement matrix $\Phi$, and the basis matrix $\Psi$, the purpose of CS reconstruction is to find $\alpha$ such that

$\|\boldsymbol{\alpha}\|_{1}$ is minimized subject to $\|\mathbf{y}-\mathbf{D} \boldsymbol{\alpha}\|_{2} \leq \varepsilon$.

Minimizing the $l_{1}$ norm of $\alpha$ amounts to minimize the number of non-zero entries of the solution $\boldsymbol{\alpha}^{\#}$ and therefore force the solution to be a sparse vector. Enforcing the constraint $\|\mathbf{y}-\mathbf{D} \boldsymbol{\alpha}\|_{2} \leq \varepsilon$ would ensure the reconstructed signal $\mathbf{x}^{\#}=\Psi \boldsymbol{\alpha}^{\#}$ will yield the same measurements $\mathbf{y}$.

In order to reconstruct signal with a desired precision, the random sampling number value $M$ needed for reconstruction, signal sparsity level $K$, and the length of reconstructed signal $N$ need to satisfy an empirical formula: $M=c_{1} \cdot K \cdot \log (N)\left(c_{1}\right.$ is an experience constant). However, in the practical application, the sparsity level of the underlying signal is unknown in advance. Therefore, we cannot choose a reasonable acquisition number $M$. Typically, one often has to collect sufficient sampling values and then do signal reconstruction. In this case the number of samples used for signal reconstruction may be far more than the theoretical sampling number required for the reconstruction. On the other hand, when we have acquired a certain number of sampling values through a certain sampling rate, considering the requirements of the sample value for storage resources and transmission bandwidth, it always wishes to store a small amount of data for signal reconstruction, It requires to reduce the redundant information in the sampling sequence.

\section{Redundancy Reduction Algorithm}

Here, we propose a coherence based redundancy reduction algorithm for RES, which will be wished to adaptively choose the acquisition number with unavailable of prior information of signal sparsity level.

Define the correlation between the every two sampling values as follows:

$$
\mu\left(y_{i}, y_{d}\right)=\sqrt{N} \square \max _{1 \leq d<i \leq M}\left|\left\langle\boldsymbol{\phi}_{l}, \boldsymbol{\phi}_{d}\right\rangle\right|
$$


where $i \neq d, \phi_{i}$ is the row vector of measurement matrix $\Phi$. Figure 3 illustrates the coherence test with respect to RES acquisition number. Note from Figure 3, with increase of the acquisition number, the coherence of the samples is increasing. Large value of coherence means that the difference between the current reconstruction and the previous reconstruction is small. On the other hand, in the traditional sequential compressive signal processing algorithm, the small reconstruction difference will stop the sampling process. However, we can find that, the coherence does not always go larger. Therefore, based on the difference between the current reconstruction and the previous reconstruction does not work for RES signal reconstruction.

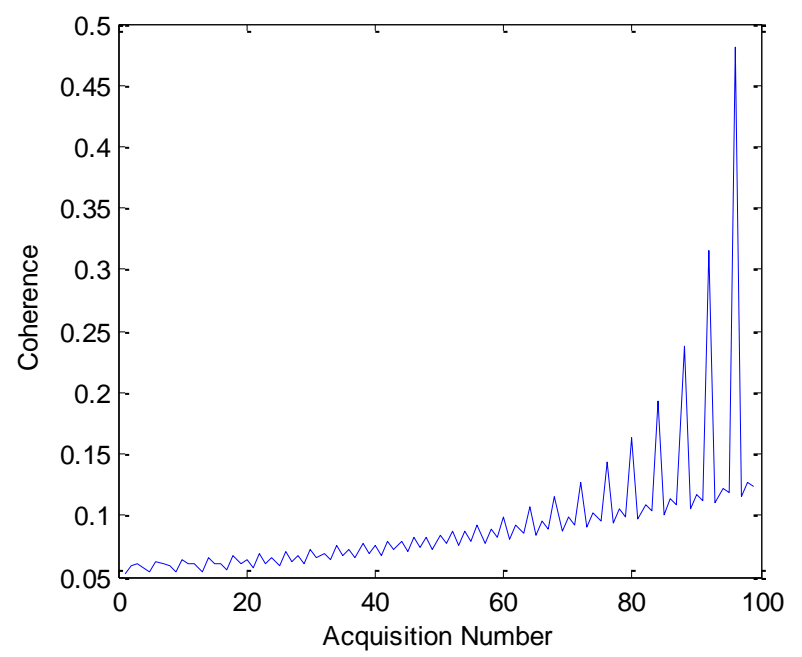

Figure 3. Coherence Test with Respect to RES Acquisition Number

According to the above analysis, we present a new redundancy reduction algorithm for RES signal reconstruction. Let $\mathbf{x}^{\#}$ be the reconstructed signal, and $\mathbf{y}_{M}$ be the sampling sequence with length of $M$, and $\mathbf{x}_{M}{ }^{\#}$ be the reconstruction from $\mathbf{y}_{M}$. After a new RES acquisition, one can obtain RES sampling sequence $\mathbf{y}_{M+1}$. Verify the correlation between $\mathbf{y}_{M+1}$ and $\mathbf{y}_{M}$, if the samples are not coherent, reconstruct $\mathbf{x}_{M+1}{ }^{\#}$ form $\mathbf{y}_{M+1}$. Otherwise, $\mathrm{y}[M+1]$ should be removed from sampling sequence. Compare $\mathbf{x}_{M+1}{ }^{\#}$ and $\mathbf{x}_{M}{ }^{\#}$, if they are close, one can make the conclusion that, $\mathbf{x}^{\#}=\mathbf{x}_{M}^{\#}$. That is the original signal can be accurately reconstructed from $M$ RES samples.

The steps of redundancy reduction algorithm are as follows:

(1) Do $M$ RES acquisitions, obtain $\mathbf{y}_{M}=\left[\mathrm{y}_{1}, \mathrm{y}_{2}, \ldots \mathrm{y}_{M}\right]^{T}$, construct measurement matrix $\boldsymbol{\Phi}$ $=\left[\phi_{1}, \phi_{2}, \ldots, \phi_{M}\right]^{T}$ based on RES random sampling time intervals, reconstruct $\mathrm{x}_{M} \#$ from $\mathbf{y}_{M}$.

(2) Do the $(M+1)$ th RES acquisition, obtain sampling sequence $\mathbf{y}_{M+1}$, construct measurement matrix. Compute coherence between the current samples and the previous samples and get a maximum one $\mu_{M+1}$. If $\mu_{M+1}<\sigma_{\mu}$ ( $\sigma_{\mu}$ is a preset constant), go to step (3), otherwise, remove y[M+1], and repeat (2).

(3) Reconstruct $\mathbf{x}_{M+1}{ }^{\#}$ from $\mathbf{y}_{M}$. Compare $\mathbf{x}_{M+1}{ }^{\#}$ with $\mathbf{x}_{M}$, if the absolute difference is smaller than $\sigma$ ( $\sigma$ is a preset threshold $)$, sampling process should be stopped, and $\mathbf{x}^{\#}=\mathbf{x}_{M}^{\#}$. Otherwise go to (2).

\section{OMP Recovery Algorithm}

To solve the optimization problem of (12), one may either apply convex programming [13] or use a family of greedy pursuit algorithm [14]. For real time RES signal reconstruction, the large computation cost of convex program makes it a less appealing 
approach. In this work, we adopt the orthogonal matching pursuit algorithm [15] (OMP), a variant of greedy pursuit algorithm to solve for the sparse vector $\boldsymbol{\alpha}$.

For convenience, denote a matrix $\mathbf{D}=\boldsymbol{\Phi} \cdot \Psi$. Equation (12) implies that the sparse solution vector $\boldsymbol{\alpha}$ should use the fewest possible columns of $\mathbf{D}$ matrix to approximate the observation $\mathbf{y}$. This can be formulated as a subset selection problem where a minimum subset of columns of $\mathbf{D}$ matrix is chosen to approximate the observation vector $\mathbf{y}$ in the least square sense. The OMP algorithm successively chooses an additional column of $\mathbf{D}$ matrix to reduce the approximation error. Equivalent, the OMP method begins with a tentative solution of $\boldsymbol{\alpha}$ with a single non-zero entry, and gradually adding non-zero entries one by one until the approximation error of $\mathbf{y}$ meets a pre-determined criterion. More specifically, denote $\mathbf{B}$ to be a matrix formed by the subset of columns of the $\mathbf{D}$ matrix whose column indices correspond to non-zero entries of the $\alpha$ vector. Then, the measurement vector $\mathbf{y}$ may be approximated by its projection onto the subspace spanned by columns of $\mathbf{B}$ :

$\hat{\mathbf{y}}=\mathbf{P}_{\mathbf{B}} \mathbf{y}=\mathbf{B}\left(\mathbf{B}^{T} \mathbf{B}\right)^{-1} \mathbf{B}^{T} \mathbf{y}=\mathbf{B B}^{\dagger} \mathbf{y}$

where $\mathbf{B}^{+}$is the Moore-Penrose pseudo-inverse of the $\mathbf{B}$ matrix. Note that the residual $\mathbf{r}=$ $\mathbf{y}-\hat{\mathbf{y}}$ is perpendicular to the subspace spanned by $\mathbf{B}$ :

$\mathbf{P}_{\mathrm{B}} \mathbf{r}=\mathbf{P}_{\mathrm{B}}(\mathbf{y}-\hat{\mathbf{y}})=\mathbf{P}_{\mathrm{B}} \mathbf{y}-\mathbf{P}_{\mathrm{B}}\left(\mathbf{P}_{\mathrm{B}} \mathbf{y}\right)=\mathbf{P}_{\mathrm{B}} \mathbf{y}-\mathbf{P}_{\mathrm{B}} \mathbf{y}=\mathbf{0}$.

Here the property of a projection matrix $\mathbf{P}_{\mathbf{B}} \cdot \mathbf{P}_{\mathbf{B}}=\mathbf{P}_{\mathbf{B}}$ is used.

Denote $\mathbf{C}$ to be a matrix whose columns are formed by the set difference of columns of the $\mathbf{D}$ matrix and those of the $\mathbf{B}$ matrix. A greedy criterion to select one more column of $\mathbf{C}$ to be moved to $\mathbf{B}$ is to choose one that has the smallest angle between $\mathbf{r}$ and itself. Let $\mathbf{c}$ and $\mathbf{c}^{\prime}$ be columns of $\mathbf{C}$, then the best choice of $\mathbf{c}$ must satisfy.

$\left\|\mathbf{P}_{\mathbf{c}} \mathbf{r}\right\|=\left\|\mathbf{r}^{T} \mathbf{c}\right\| /\|\mathbf{c}\| \geq\left\|\mathbf{r}^{T} \mathbf{c}^{\prime}\right\| /\left\|\mathbf{c} \mathbf{c}^{\prime}\right\|=\left\|\mathbf{P}_{\mathbf{c}^{\prime}} \mathbf{r}\right\|$.

Once the new $\mathbf{c}$ is chosen, the $\mathbf{B}$ matrix and $\mathbf{C}$ matrix will be updated, and the residue vector can be updated. The algorithm will be stopped when the norm of $\mathbf{r}$ is smaller than the preset threshold $\varepsilon$. The $\alpha$ vector then has the form of

$\boldsymbol{\alpha}=\left[\begin{array}{c}\mathbf{B}^{\dagger} \mathbf{y} \\ \mathbf{0}_{(N-K) \times 1}\end{array}\right]$.

\section{Experiment}

In this section, several experiments are reported to investigate the proposed algorithm. We define the length of reconstructed waveform $N=250$, then the equivalent sampling rate $f_{e}$ and the sampling rate $f_{s}$ satisfy $f_{e}=N \cdot f_{s}$. In all the experiments, signal is reconstructed using OMP. Define signal to noise ratio (SNR) :

$S N R=20 \cdot \log _{10}\left(\frac{\square \mathrm{x} \square}{\square \mathrm{x}-\mathrm{x}^{\#} \square}\right)$

where $\mathbf{x}$ is the original signal, and $\mathbf{x}^{\#}$ is the reconstructed signal. 


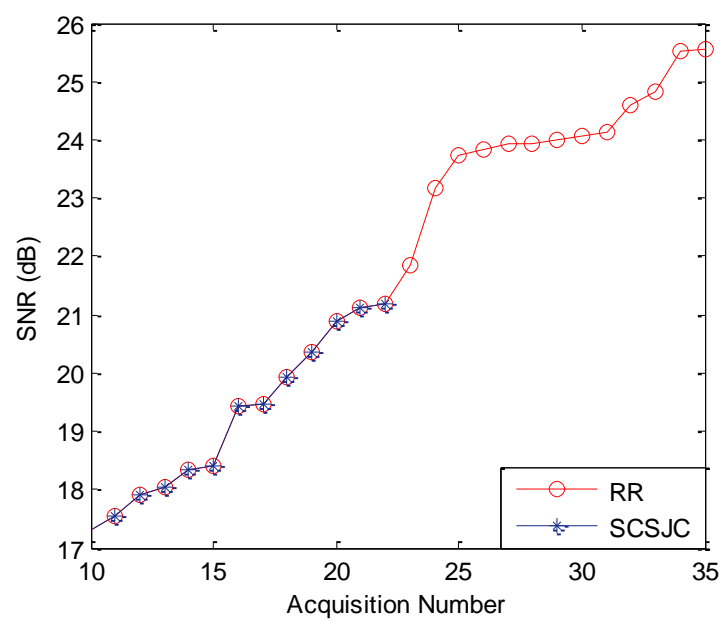

\section{Figure 4. Comparison of Reconstruction Performance via the Original Method and the Improved Method}

The first experiment compares the proposed algorithm and the traditional sequential compressive signal processing algorithm. Figure 4 depicts the comparison. If the traditional sequential is employed, $M=21$ meets the sampling stop criteria, it yields $\mathrm{SNR}=14.2 \mathrm{~dB}$ (referred as "SCSJC"). However, with the proposed algorithm, one can find that the coherence is large, RES sampling should continue. Until $M=35$, the samples yield a small coherence and the reconstruction difference is small, the sampling process is stopped. Reconstruction achieves $\mathrm{SNR}=17.1 \mathrm{~dB}$ (referred as "RR"). Clearly, the accuracy of reconstructed waveform has been improved.

In the second experiment, a sinusoidal signal is acquired with RES system and reconstructed. Figure 5(a) shows the reconstruction using the traditional compressive processing criteria, The criteria makes the decision that the signal reconstruction only needs 33 RES samples, and reconstruction yields SNR $=27.4 \mathrm{~dB}$. Figure $5(\mathrm{~b})$ depicts the reconstruction using the proposed redundancy reduction algorithm, The criteria makes the decision that the signal reconstruction needs 42 RES samples, and reconstruction yields $\mathrm{SNR}=30.8 \mathrm{~dB}$.

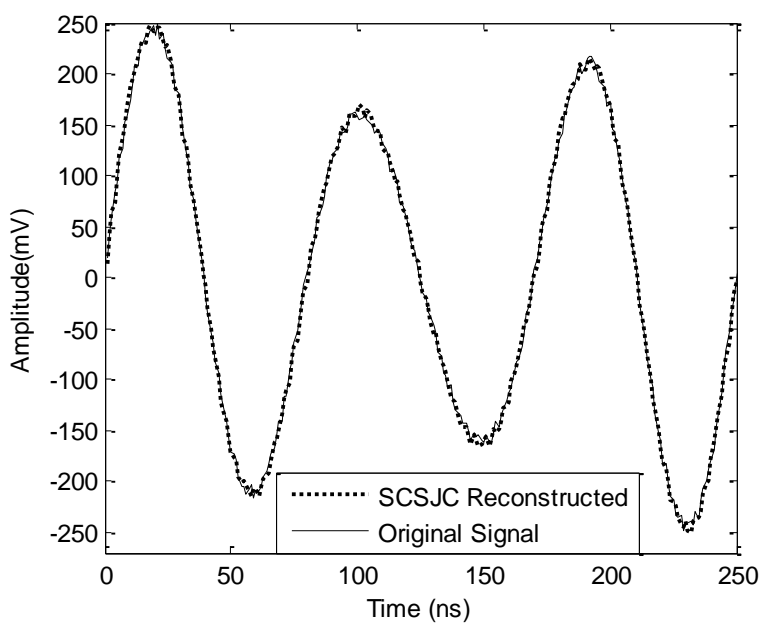

(a) 


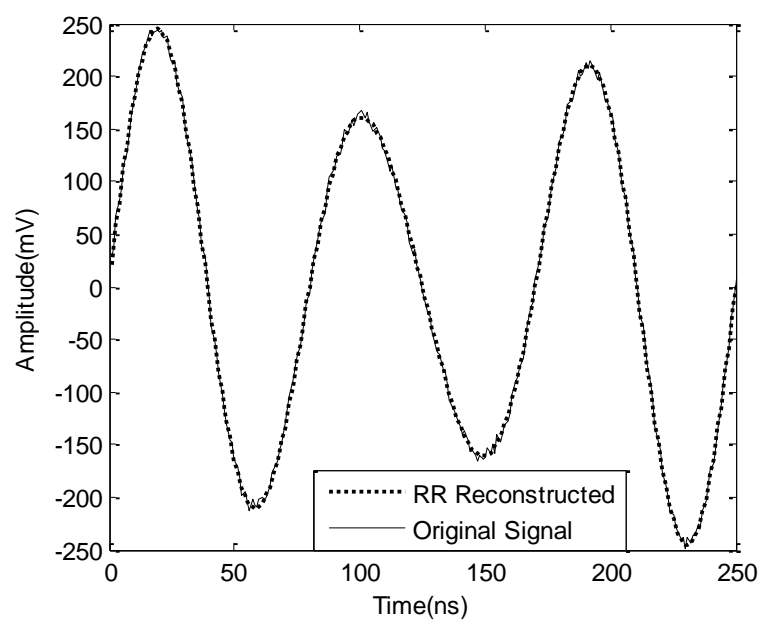

(b)

Figure 5. Sinusoidal Signal Reconstruction. (a) Based on Traditional Criteria. (b) Based on the Proposed Criteria

Finally, a square signal is acquired with RES system and reconstructed. Figure 6(a) shows the reconstruction using the traditional compressive processing criteria, The criteria makes the decision that the signal reconstruction only needs 89 RES samples, and reconstruction yields SNR $=14.2 \mathrm{~dB}$. Figure 6(b) depicts the reconstruction using the proposed redundancy reduction algorithm. The criteria makes the decision that the signal reconstruction needs 98 RES samples, and reconstruction yields $\mathrm{SNR}=16.8 \mathrm{~dB}$. Since the square signal is less sparse than the sinusoidal signal, more samples needs to reconstruct the square signal.

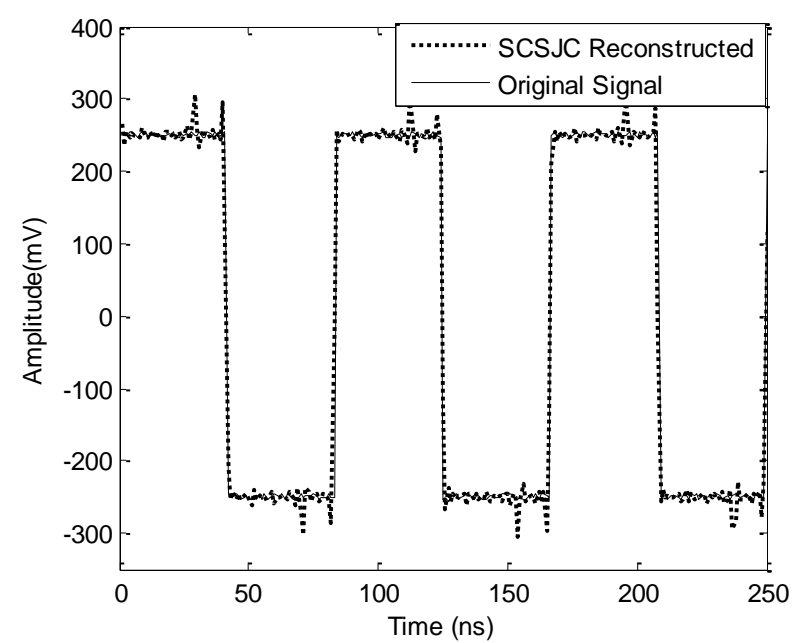

(a) 


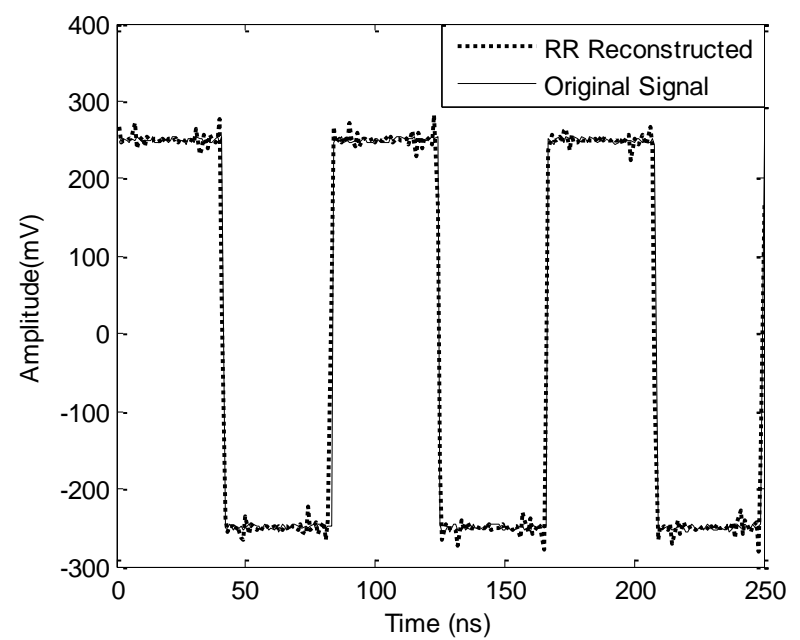

(b)

Figure 6. Square Wave Reconstruction. (a) Based on Traditional Criteria. (b) Based on the Proposed Criteria

\section{Conclusion}

CS based signal reconstruction algorithm can improve the RES signal reconstruction. This paper proposed a redundancy reduction algorithm to mitigate the performance of CSRES reconstruction to the unknown sparsity level. Difference from the traditional sequential compressive signal processing criteria, the proposed algorithm combines the sampling coherence and the reconstruction difference. Only the sampling sequence with small coherence is reconstructed, and then the current reconstruction and previous reconstruction are compared. The experimental results demonstrate the feasibility of the proposed algorithm. With the help of proposed algorithm, higher SNR could be achieved.

\section{Acknowledgements}

This work is supported in part by the National Natural Science Foundation of China (Grant No. 61301264), in part by the Ph.D. Programs Foundation of Ministry of Education of China (Grant No. 20130185120019), and in part by the Fundamental Research Funds for the Central Universities (Grant No. ZYGX2013J089).

\section{References}

[1] F. M. C. Clemencio, C. F. M. Loureiro and C. M. B. Correia, "An Easy Procedure for Calibrating Data Acquisition Systems Using Interleaving," IEEE Trans. Nuclear Science, vol. 54, no. 4, (2007), pp. 1227-1231,

[2] V. G. Ivchenko, A. N. Kalashnikov, R. E. Challis and B. R. Hayes-Gill, "High-Speed Digitizing of Repetitive Waveforms Using Accurate Interleaved Sampling," IEEE Trans. Instrum. Meas., vol. 56, no. 4, (2007),pp. 1322-1328.

[3] R. A. Witte, "Sample Rate and Display Rate in Digitizing Oscilloscopes," Hewlett-Packward Journal, vol. 43, no. 1, , (1992) Feb, pp. 18-19/

[4] P. J. Pupalaikis, "Random Interleaved Sampling", [Online]. Available: http://www.lecroy.com/files/ WhitePapers/WP_Ris_102203.pdf

[5] P. D. Hale, C. M. Wang, D. F. Williams, K. A. Remley and J. D. Wepman, "Compensation of Random and Systematic Timing Errors in Sampling Oscilloscopes," IEEE Trans. Instrum. Meas., vol. 55, no. 6, (2006), pp. 2146-2154.

[6] Y. Zhao, X. Zhuang and L. Wang, "The Research and Application of Random Sampling in Digital Storage Oscilloscope," IEEE Circuit and Systems International Conference, Chengdu, China, (2009), April 28-29. 
[7] D. Donoho, "Compressed sensing," IEEE Trans. Inform. Theory, vol. 52, (2006), pp. 1289-1306.

[8] E. Candès, J. Romberg and T. Tao, "Robust uncertainty principles: Exact signal reconstruction from highly incomplete frequency information," IEEE Trans. Inform. Theory, vol. 52, (2006), pp. 489-509.

[9] E. Candès and M. Wakin, "An introduction to compressive sampling [a sensing/sampling paradigm that goes against the common knowledge in data acquisition]," IEEE Sig. proc. Mag., vol. 52, no. 2, (2006), pp. 21-30,

[10] Y. J. Zhao, Y. H. Hu and H. J. Wang, "Enhanced Random Equivalent Sampling Based on Compressed Sensing, " IEEE Trans. Instrumentation and Measurement. vol. 61, no. 3, (2012), pp. 579-586.

[11] D. M. Malioutov, S. R. Sanghavi, and A. S. Willsky, "Sequential compressed sensing," IEEE Journal of Selected Topics in Signal Processing vol. 4, no. 2, (2010), pp. 435-444.

[12] A. C. Gilbert, M. J. Strauss and J. A. Tropp, "Improve time bounds for near-optimal sparse Fourier representation via sampling," in Proc. Wavelets XI at SPIE Optics and Photonics, San Diego, CA, (2005).

[13] J. A. Tropp, "Algorithms for simultaneous sparse approximation. Part I: Convex relaxation," Signal Process. (Special Issue on Sparse Approximations in Signal and Image Processing), vol. 86, (2006), pp. 589-602.

[14] J. A. Tropp, "Algorithms for simultaneous sparse approximation. Part II: Greedy pursuit," Signal Process. (Special Issue on Sparse Approximations in Signal and Image Processing), vol. 86, (2006), pp. $572-588$.

[15] J. A. Tropp and A. C. Gilbert, "Signal recovery from random measurements via orthogonal matching pursuit," IEEE Trans. Inform. Theory, vol. 53, no. 12, (2007), pp. 4655-4666.

[16] Z. Lv, A. Tek, F. D. Silva, C. E.-Mot, M. Chavent and M. Baaden, "Game on, science-how video game technology may help biologists tackle visualization challenges", PloS one 8, no. 3, (2013), e57990.

[17] Yu Song, "A Rapid and High Reliable Identify Program for Nighttime Pedestrians", Infrared Physics \& Technology, (2015).

[18] D. Jiang, Z. Xu, W. Li and Z. Chen, "Network Coding-Based Energy-Efficient Multicast Routing Algorithm for Multi-hop Wireless Networks", Journal of Systems and Software, (2015) March 12.

[19] J. Yang, “ Objective Evaluation Criteria for Stereo Camera Shooting Quality under Different Shooting Parameters and Shooting Distances. Sensors Journal, IEEE, vol. PP, no.99, pp.1-1

[20] X. Li, Z. Lv, B. Zhang, L. Yin, W. Wang, S. Feng, and J. Hu, "Traffic Management and Forecasting System Based on 3D GIS", 2015 15th IEEE/ACM International Symposium on Cluster, Cloud and Grid Computing (CCGrid). IEEE, (2015).

[21] K. Leng, “ Designing of a I-shaped less-than-truckload cross-dock: A simulation experiments study. International Journal of Bifurcation and Chaos, (2015).

[22] Z. Lv, "Wearable Smartphone: Wearable Hybrid Framework for Hand and Foot Gesture Interaction on Smartphone", IEEE International Workshop on Wearable Computer Vision Systems at International Conference on Computer Vision 2013 (ICCV 2013), Sydney, Australia, (2013).

[23] D. Jiang, Z. Xu, P. Zhang and T. Zhu, "A transform domain-based anomaly detection approach to network-wide traffic", Journal of Network and Computer Applications, vol. 40, (2014), pp. 292-306.

[24] L. Zhang, B. He, J. Sun, M. Lai and Z. Lv, "Double Image Multi-Encryption Algorithm based on Fractional Chaotic Time Series", Journal of Computational and Theoretical Nanoscience, (2016).

[25] W. Luo, Z. Wang, Z. LV, "Method to Acquire a Complete Road Network in High-resolution Remote Sensing Image Based on Tensor Voting Algorithm", EXCLI JOURNAL, vol.14, (2015) Nov.

[26] Y. Geng, J. Chen, R. Fu, G. Bao and K. Pahlavan, "Enlighten wearable physiological monitoring systems: On-body rf characteristics based human motion classification using a support vector machine", IEEE transactions on mobile computing, vol. 1, no. 1, (2015) Apr., pp. 1-15,

[27] J. Hu and Z. Gao, "Distinction immune genes of hepatitis-induced heptatocellular carcinoma", [J]. Bioinformatics, vol. 28, no. 24, (2012), pp. 3191-3194.

[28] J.Hu and Z. Gao, "Modules identification in gene positive networks of hepatocellular carcinoma using Pearson agglomerative method and Pearson cohesion coupling modularity", [J]. Journal of Applied Mathematics, (2012).

[29] J. He, Y. Geng, F. Liu and C. Xu, "CC-KF: Enhanced TOA Performance in Multipath and NLOS Indoor Extreme Environment”, IEEE Sensor Journal, vol. 14, no. 11, , pp. 3766-3774.

[30] S. Zhou, L. Mi, H. Chen and Y. Geng, "Building detection in Digital surface model", 2013 IEEE International Conference on Imaging Systems and Techniques (IST), (2012) October.

[31] J. He, Y. Geng and K. Pahlavan, "Toward Accurate Human Tracking: Modeling Time-of-Arrival for Wireless Wearable Sensors in Multipath Environment", IEEE Sensor Journal, vol. 14, no. 11, (2014) Nov, pp. 3996-4006.

[32] J. Hu, Z. Gao and W. Pan, "Multiangle Social Network Recommendation Algorithms and Similarity Network Evaluation", [J]. Journal of Applied Mathematics, (2013). 


\section{Authors}

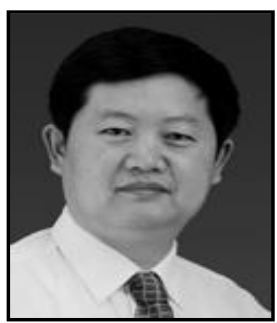

Jianguo Huang, he has born in Jiangsu, China in 1962. He received his B.S. degree in University of Electronic Science and Technology of China (UESTC), Chengdu, China. Now he is a professor in School of Automation Engineering, UESTC. His current research interests include radar signal processing, testing and instrument. 
International Journal of Signal Processing, Image Processing and Pattern Recognition Vol. 9, No. 5 (2016) 